\title{
Preferencias turísticas de los adultos mayores de la región Callao
}

Artículos originales: GEOGRAFÍA

Recibido: 25/04/2021

Aprobado: 25/06/2021

Publicado: 14/10/2021
María del Carmen More Altuna

Universidad Nacional Mayor de San Marcos mmorea@unmsm.edu.pe

\begin{abstract}
RESUMEN
En la región Callao se está produciendo un lento proceso de envejecimiento demográfico, es decir, los adultos mayores están en constante crecimiento, alcanzando el $12.4 \%$ de la población total de la región. Asimismo, la esperanza de vida aumentó a 78.4 ańos. Generalmente, la situación laboral de esta población es cesante o jubilada. Por tanto, está en una etapa de su vida en la que dispone de tiempo libre, que podría utilizar para realizar viajes turísticos o actividades recreativas, acciones que beneficiarían su salud y mejorarían su calidad de vida. En el país, los adultos mayores viven una situación de marginación y descuido. Debido a ello, deberían ser incorporados dentro de las políticas públicas de turismo, que conllevaría a la elaboración de planes turísticos especializados para este grupo, considerando sus preferencias turísticas, motivaciones, capacidad económica, su estado de salud, entre otros aspectos. Es ineludible considerar la incorporación de la población adulta mayor al marketing turístico, favoreciendo económicamente a las agencias turísticas, especialmente en temporada baja y durante todo el año. Para conocer las preferencias turísticas de este segmento poblacional se aplicó una encuesta a 384 personas que conforman la muestra representativa de la población adulta mayor residente en la región Callao.
\end{abstract}

Palabras clave: Envejecimiento demográfico, adulto mayor, preferencias turísticas, turismo y recreación, región Callao.

\section{Tourist preferences of older adults from Callao region}

\begin{abstract}
In Callao region a slow process of demographic aging is taking place, that is, older adults are constantly growing, reaching $12.4 \%$ of the total population of the region. Furthermore, life expectancy has increased to 78.4 years. Generally, the employment situation of this population is unemployed or retired. Therefore, they are at a stage in your life in which they have free time, which they could use for tourist trips or recreational activities, actions that would benefit their health and improve their quality of life. In the country, the elderly live in a situation of marginalization and neglect. Due to this, they should be incorporated into tourism public policies, which would lead to the elaboration of specialized tourist plans for this group, considering their tourist preferences, motivations, economic capacity, their state of health, among other aspects. It is unavoidable to consider incorporating the older adult population into tourism marketing, economically favoring tourism agencies, especially in low season and throughout the year. To know the tourist preferences of this population segment, a survey was applied to 384 people who make up the representative sample of the older adult population residing in Callao region.
\end{abstract}

KeYwords: Demographic aging, elderly, tourist preferences, tourism and recreation, Callao region. 


\section{Introducción}

E l presente trabajo estudia temas relacionados con aspectos turísticos de la población adulta mayor residente en la región Callao. El turismo es una actividad que produce efectos económicos positivos en los lugares donde se fomenta y desarrolla esta actividad. Teniendo en consideración que el Callao, por su posición estratégica en la cuenca del Pacífico Sur, está destinado a convertirse en el centro del comercio internacional y en el centro principal de servicios portuarios y turísticos de gran jerarquía tanto a nivel nacional como internacional, es ineludible efectuar acciones para promover el turismo, en base a un modelo de desarrollo turístico sostenible. Si consideramos que en la región la población de 60 y más años de edad se incrementa constantemente, así como la esperanza de vida, sería conveniente promocionar el turismo e incorporar al marketing turístico a los adultos mayores, de modo que las agencias de turismo podrían beneficiarse económicamente tanto en temporada baja como durante todo el año.

En tanto, la Organización de las Naciones Unidas considera que una sociedad envejece cuando más del $7 \%$ de la población supera los 60 años de edad. Según los Censos Nacionales 2017, la población adulta mayor es de 123157 habitantes, representando el 12.4 $\%$ de la población total de la región Callao.

En Madrid, en el año 2002, durante la Segunda Asamblea Mundial sobre Envejecimiento, se reiteró la necesidad de hacer "cambios en las actitudes, las políticas y las prácticas a todos los niveles y en todos los sectores, para que puedan concretarse las enormes posibilidades que brinde el envejecimiento del siglo XXI». Además, el plan recomienda el bienestar social que incluye incentivos de recreación y el mejor aprovechamiento del ocio de los adultos mayores. En el foro sobre «Viajes y Tercera Edad», se refirió a los beneficios de dichos programas tales como sacar a los adultos mayores del aislamiento, luego de jubilarse, devolverles el placer de desplazarse para preservar el mayor tiempo posible, tanto su independencia física como social y brindarles la posibilidad de visitar lugares y ofrecerles mayor libertad.

Por lo general, la población de adultos mayores se encuentra en situación de cesante o jubilado; en tal sentido, disponen de mayor tiempo libre y viven una vida sedentaria que perjudica su salud. Por consiguiente, se recomienda que este grupo poblacional practique alguna actividad física como realizar viajes turísticos o actividades recreativas que los ayudaría a contrarrestar los efectos nocivos del sedentarismo y así conseguir una mejor salud y calidad de vida. Por lo tanto, es de gran importancia la participación de este grupo poblacional en la sociedad, disponiendo del tiempo libre que poseen para dedicarlo al turismo y a la recreación.

Por otro lado, en el país la población adulta mayor atraviesa por una situación de marginación e indiferencia, que afecta su ámbito social, económico, cultural y de salud. Es en este contexto que el Estado y las diferentes instituciones relacionadas con el turismo deberían incorporar a este segmento poblacional dentro de las políticas públicas de turismo, a fin de establecer estrategias orientadas a elaborar planes turísticos especializados para este grupo, considerando sus preferencias turísticas, motivaciones, percepciones, deseos, capacidad económica, su estado de salud, edad, disponibilidad de tiempo, entre otros aspectos.

\section{Relación del estudio con otros similares}

\section{Internacionales}

Al revisar la literatura sobre la actividad turística de los adultos mayores, se encontraron los siguientes documentos que a continuación se describen:

En el año 2009, en México, los autores María de los Ángeles López Nórez, Francisco J. Llera Pacheco y Patricia Vásquez Ramírez, publicaron el trabajo titulado El Papel del turismo en la Recreación del Adulto Mayor en México.

El estudio trata de la forma en que las personas adultas mayores utilizan su tiempo libre, los beneficios de la utilización adecuada del tiempo libre y como la recreación puede contribuir al desarrollo humano de los adultos mayores. Asimismo, indica que el turismo es un medio para mejorar la calidad de vida de este segmento de la población.

Cueva (2014) en su estudio exploratorio - -descriptivo del segmento adulto mayor- su oferta turística y diseño de estrategias de intervención en la ciudad de Quito, señala que el estudio tiene la intención de identificar sus gustos y preferencias para es- 
tablecer estrategias de intervención en actividades de turismo dentro y fuera del Ecuador, ya que cuentan con tiempo libre y la necesidad de mantenerse activos y saludables.

\section{Nacionales}

Castillo (2018) desarrolla un análisis de las experiencias en socialización y gestión turística en el adulto mayor valente en Lima, para lo cual propone un modelo de gestión para turismo especializado en este tipo de turistas, el cual está enfocado en las personas de 60 años a más, que tienen posibilidades de socializar a través de la práctica del turismo que se asocian a las preferencias turísticas y a la capacidad económica del adulto mayor.

\section{Justificación de la investigación}

Teniendo en consideración que los adultos mayores constituyen un grupo poblacional que está en constante crecimiento en la región Callao, que en los próximos años la esperanza de vida se incrementará de manera importante, y que en esta etapa de su vida, por tener tiempo disponible, encuentran la mejor oportunidad para realizar viajes turísticos y actividades recreativas, el presente estudio de investigación se justifica porque nos permitirá conocer la actividad turística y las preferencias turísticas de la población adulta mayor residente en la región Callao. Conjuntamente, se pretende que los resultados obtenidos puedan ser utilizados para diseñar programas turísticos que conlleven a un desarrollo sostenido de la actividad turística de los adultos mayores, considerando una oferta diversificada, con implementación de circuitos turísticos, con paquetes de viajes económicos que ofrezcan servicios turísticos especializados para este grupo etario.

\section{Objetivo de la investigación}

\section{Objetivo general}

Determinar la manera en que se relaciona el proceso de envejecimiento demográfico y el nivel de preferencias turísticas de la población adulta mayor de la región del Callao.

\section{Marco teórico}

\section{Región Callao}

La Provincia Constitucional del Callao fue elevada a la categoría de Región durante el Gobierno del presidente Alejandro Toledo, mediante Ley $\mathrm{N}^{\circ} 27867$ el 1 de enero de 2003. La región Callao se ubica en la parte central y occidental del Perú. Presenta una excelente posición geoestratégica en la cuenca del Pacífico Sur. Adicionalmente, en su territorio se encuentran las instalaciones del puerto del Callao, principal puerto del Perú, y el aeropuerto internacional Jorge Chávez, convirtiéndose en la principal puerta de entrada y de salida de personas y de bienes económicos. Según los resultados del censo 2017, el $100 \%$ del territorio de la región es área urbana. Esta situación es resultado del proceso de expansión urbana ocurrida en la región.

La región Callao es considerada como el segundo centro industrial del país, después de Lima, en donde se concentran diversas empresas manufactureras. Por ello, las actividades predominantes son la industria y las que están vinculadas al sector terciario. Cabe destacar que la región Callao cuenta con diversos atractivos turísticos tanto naturales como culturales, ideal para incentivar la actividad turística en la región. En los últimos años, la región se ha caracterizado por su gran actividad industrial, comercial y residencial.

\section{Envejecimiento demográfico}

El envejecimiento es un proceso que se está desarrollando en la región Callao, ocasionando el aumento de la población adulta mayor. El envejecimiento poblacional se debe a dos factores. En primer lugar, a la disminución de la natalidad que se manifiesta en el aumento de la población adulta mayor y en una disminución de la población menor de 15 años; y, en segundo lugar, a la disminución de la tasa de mortalidad, que incide en el aumento de la esperanza de vida de las personas (Cornachione, 2006).

Por otro lado, el envejecimiento ocasiona modificaciones en los aspectos sociales, económicos y de salud. Asimismo, el envejecimiento produce cambios demográficos que están relacionados con la estructura por edad de la población, con la transición demo- 
gráfica y con la transición epidemiológica, causando variaciones en la mortalidad, morbilidad y en la esperanza de vida.

\section{Adulto mayor}

Es un segmento de la población compuesta por personas de 60 y más años de edad. Según el INEI, la población adulta mayor que reside en la región Callao se está incrementando constantemente. De acuerdo con los resultados del censo 2007, la población fue de 80909 personas, representando el 9.2\% de la población total. Los datos del censo 2017 revelan que la población adulta mayor aumentó a 123157 personas, que significan el $12.4 \%$ de la región Callao. Este aumento fue motivado por el incremento de la esperanza de vida y la disminución de la tasa de natalidad. Según Cornachione (2006), el aumento de la esperanza de vida debe ir acompańado de una adecuada calidad de vida para que esta población logre una longevidad satisfactoria en todos los aspectos de su vida. Este grupo etario experimenta cambios físicos, psíquicos, biológicos y sociales. Su salud se ve resquebrajada, adolece de enfermedades no trasmisibles o crónicas degenerativas y se ven obligados a tomar medicamentos de por vida.

\section{Preferencias turísticas}

El turismo tiene una gran connotación sicológica que se debe tener en consideración cuando se estudia el comportamiento del turista, abordando temas como la motivación, las actitudes, percepciones, deseos, preferencias turísticas, etc. Las preferencias turísticas están relacionadas con la idiosincrasia del turista. El turista puede ser definido dentro del modelo psicográfico de personalidad turística. Según este modelo, existen personalidades con características diferentes y se puede clasificar a la población en dos polos: alocéntrico y psicocéntrico. También están el casi alocéntrico, midcéntrico y semipsicocéntrico. Cada una de estas personalidades prefiere experiencias vacacionales independientes del mercado de turismo de masas. De acuerdo con la personalidad turística, las preferencias turísticas son diferentes (Plog, 1972; citado por Castaño, 2005).

\section{Turismo y recreación}

Hay diversas definiciones de turismo. Algunas son extensas y otras, más corta como la de Ramos (2016) que señala al turismo como la actividad humana que satisface diversas motivaciones del turista, las que son variadas y tienen carácter personal. El turismo debe ser específico, social, económico y humano. Los desplazamientos humanos le conceden al turista la posibilidad de deleitarse en su travesía de numerosos paisajes, creando en él diversos eventos en su conformación de nuevas experiencias de orden turístico. Para hacer turismo se debe considerar, entre otros aspectos, los deseos, los anhelos y las expectativas del turista.

En cambio, otros autores consideran en sus definiciones otros aspectos como De la Torre (1980) que se refiere al turismo como la actividad que consiste en el traslado voluntario y temporal de individuos o grupos de personas con motivo de recreación, descanso, cultura o salud, y que no realizan ninguna actividad remunerada.

El turismo genera diversos efectos en lo económico, social, cultural, en el medio ambiente y en la salud de los turistas. Desde el punto de vista económico, muchos países optaron por el turismo para potenciar su desarrollo económico, en virtud de los efectos benéficos que ofrece, ya que contribuye en la balanza de pagos, en la creación de empleos y en el impacto positivo que tiene sobre otras ramas de la actividad económica (Acerenza, 2006).

La recreación al igual que el turismo están relacionados con el tiempo libre, el mismo que los adultos mayores pueden aprovechar para realizar actividades agradables, distraídas y entretenidas, por un tiempo que no exceda las 24 horas

El tiempo libre permite al individuo practicar actividades que satisfacen sus necesidades secundarias como el descanso, diversión y desarrollo personal, estas actividades pueden ser física, intelectual, deportivas, sociales, culturales (Rodríguez, 2010).

La recreación tiene que ver con educación, con abrir espacios, con crear espacios de discusión y análisis, con formar integralmente al ser humano, con educación permanente y además con diversión y con placer (Ziperovich, 2006). 


\section{Resultados}

En la región Callao se aplicó 384 encuestas a personas adultas mayores y se obtuvieron los siguientes resultados:

Con relación a la edad, vemos que el 31.0\% de los adultos mayores conforman el grupo de edad de 60 a 64 años, el $24.0 \%$ corresponden al grupo de 65 a 69 ańos, el $17.4 \%$ tienen 70 a 74 ańos, el $12.1 \%$ tienen 75 a 79 ańos, el $8.3 \%$ corresponden al grupo de 80 a 84 años y el $7.1 \%$ tienen de 85 a más años (cuadro 1).

Los resultados en cuanto al sexo de los adultos mayores muestran que el $53.3 \%$ son mujeres en tanto que el $46.7 \%$ restante son varones.

El índice de masculinidad (IM) de los adultos mayores varía. En el grupo de edad de 60 a 64 años, el IM es de 87.2 por cada 100 mujeres; en el grupo de 65 a 69 años, 88.5 por cada 100 mujeres; en el grupo de 70 a 74 ańos, $91.6 \%$ por cada 100 mujeres; en el grupo de 75 a 79 ańos, $92.3 \%$ por cada 100 mujeres; en el grupo de 80 a 85 ańos, 85.5 por cada 100 muje- res; y en el grupo de edad de 85 a más años, 72.5 por cada 100 mujeres (gráfico 1).

En la pirámide poblacional de la región Callao se observa el incremento constante de la población adulta mayor, que en cifras absolutas es de 123157 personas, representando el $12.4 \%$ de la población total de la región (cuadro 2, gráfico 2).

\section{Cuadro 1}

Región Callao: Población adulta mayor por grupo de edad, por sexo e índice de masculinidad, 2017

\begin{tabular}{|c|c|c|c|c|}
\hline $\begin{array}{c}\text { Grupo de } \\
\text { edad }\end{array}$ & Total & Hombres & Mujeres & I.M. \\
\hline Total & 123,157 & 57,510 & 65,647 & 87.6 \\
\hline 60 a 64 & 38,238 & 17,817 & 20421 & 87.2 \\
\hline 65 a 69 & 29,557 & 13,874 & 15683 & 88.5 \\
\hline 70 a 74 & 21,487 & 10,272 & 11215 & 91.6 \\
\hline 75 a 79 & 14,934 & 7,170 & 7764 & 92.3 \\
\hline 80 a 84 & 10,192 & 4,699 & 5493 & 85.5 \\
\hline 85 a más & 8,749 & 3,678 & 5071 & 72.5 \\
\hline
\end{tabular}

Fuente: INEI - Censos Nacionales 2017; XII de Población, VII de Vivienda y III de Comunidades Indígenas.

Elaboración propia.

\section{GrÁfico 1}

Región Callao: Índice de masculinidad por grupo de edad de la población adulta mayor, 2017

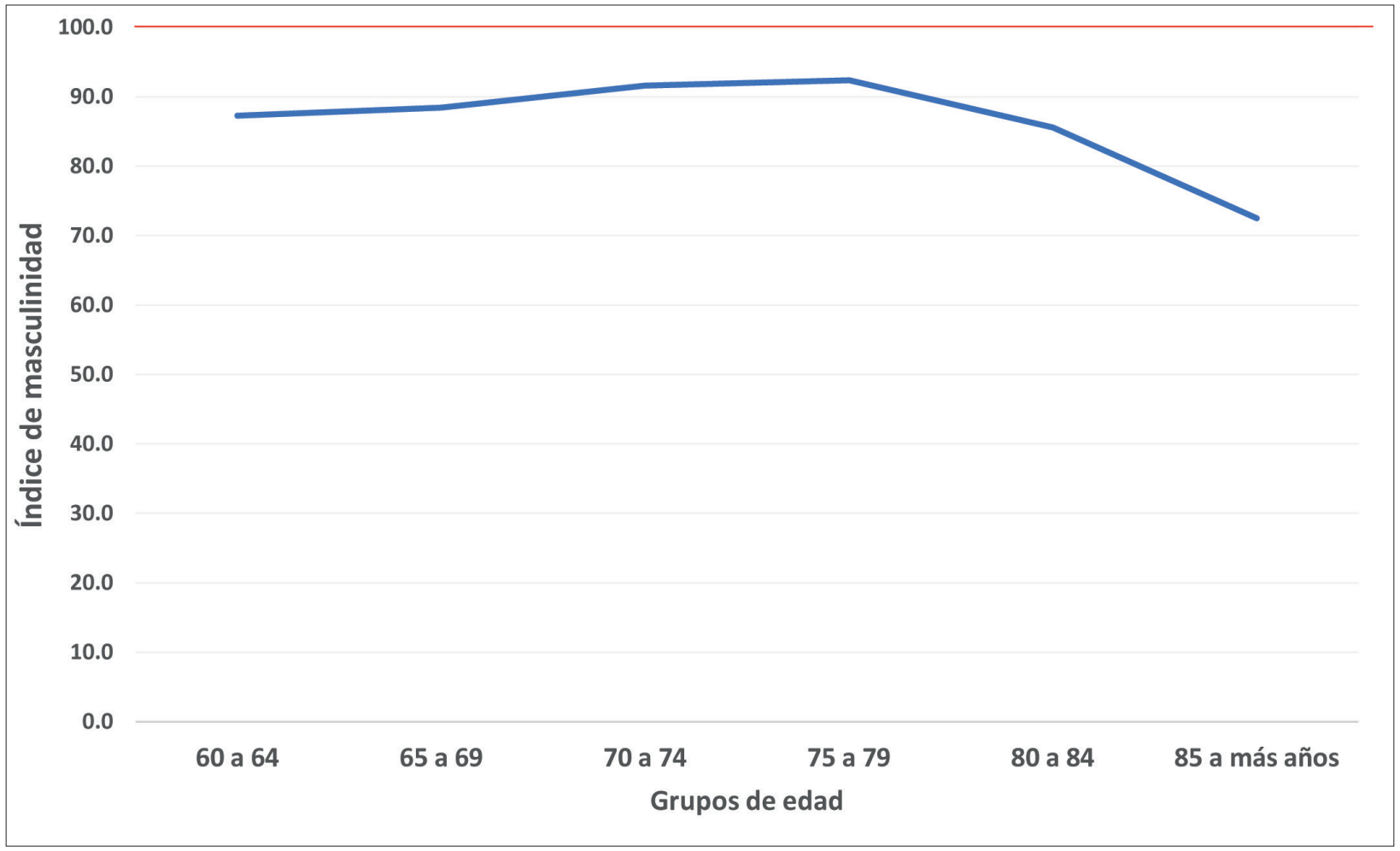

Fuente: INEI - Censos Nacionales 2017; XII Población, VII Vivienda y III de Comunidades Indígenas.

Elaboración propia 
Cuadro 2

Región Callao: Población censada por sexo, según grupo de edad, 2017

\begin{tabular}{|c|c|c|c|c|c|}
\hline $\begin{array}{c}\text { Grupo de } \\
\text { edad }\end{array}$ & Total & Hombres & $\%$ & Mujeres & $\%$ \\
\hline Total & 994494 & 485782 & 48.8 & 508712 & 51.2 \\
\hline $0-4$ & 78810 & 40359 & 4.0 & 38451 & 3.9 \\
\hline $5-9$ & 83938 & 42626 & 4.3 & 41312 & 4.2 \\
\hline $10-14$ & 80477 & 40672 & 4.1 & 39805 & 4.0 \\
\hline 15-19 & 76680 & 38601 & 3.9 & 38079 & 3.8 \\
\hline $20-24$ & 87191 & 43232 & 4.3 & 43959 & 4.4 \\
\hline $25-29$ & 82434 & 40292 & 4.1 & 42142 & 4.2 \\
\hline $30-34$ & 77898 & 37563 & 3.8 & 40335 & 4.1 \\
\hline $35-39$ & 75687 & 36332 & 3.6 & 39355 & 4.0 \\
\hline $40-44$ & 69962 & 33651 & 3.4 & 36311 & 3.7 \\
\hline $45-49$ & 60415 & 28969 & 2.9 & 31446 & 3.2 \\
\hline $50-54$ & 52565 & 24787 & 2.5 & 27778 & 2.7 \\
\hline $55-59$ & 45280 & 21188 & 2.1 & 24092 & 2.4 \\
\hline $60-64$ & 38238 & 17817 & 1.8 & 20421 & 2.1 \\
\hline $65-69$ & 29557 & 13874 & 1.4 & 15683 & 1.6 \\
\hline $70-74$ & 21487 & 10272 & 1.0 & 11215 & 1.1 \\
\hline $75-79$ & 14934 & 7170 & 0.7 & 7764 & 0.8 \\
\hline $80-84$ & 10192 & 4699 & 0.5 & 5493 & 0.5 \\
\hline 85 a más & 8749 & 3678 & 0.4 & 5071 & 0.5 \\
\hline
\end{tabular}

Fuente: INEI - Censos Nacionales 2017; XII Población, VII Vivienda y III de Comunidades Indígena.

Elaboración propia

En cuanto al distrito donde reside, se observa que el $50.8 \%$ de los encuestados viven en el Cercado del Callao; el 19.0\%, en Ventanilla; el $11.7 \%$, en Bellavista; el 10.2\%, en La Perla; el 4.7\%, en Carmen de la Legua Reynoso; el 2.1\%, en Mi Perú; y el 1.0\% reside en La Punta (cuadro 3, gráfico 3).

Los datos del estado civil señalan que el $8.1 \%$ son solteros; el $57.0 \%$, casados; el $21.5 \%$, viudos; el $6.0 \%$, divorciados; el $7.0 \%$, convivientes; y el $0.5 \%$, separados (cuadro 4, gráfico 4).

Con relación al nivel educativo, las cifras demuestran que el $1.6 \%$ de los encuestados no tienen nivel educativo; el $18.2 \%$ tienen primaria, el $49.7 \%$ poseen nivel secundaria y el $30.5 \%$ tienen estudios superior universitario y no universitario (cuadro 5, gráfico 5).

Con respecto a la interrogante sobre la situación laboral, el $33.9 \%$ de la muestra son trabajadores acti-
GráfICo 2

Región Callao: Pirámide poblacional, según censo 2017

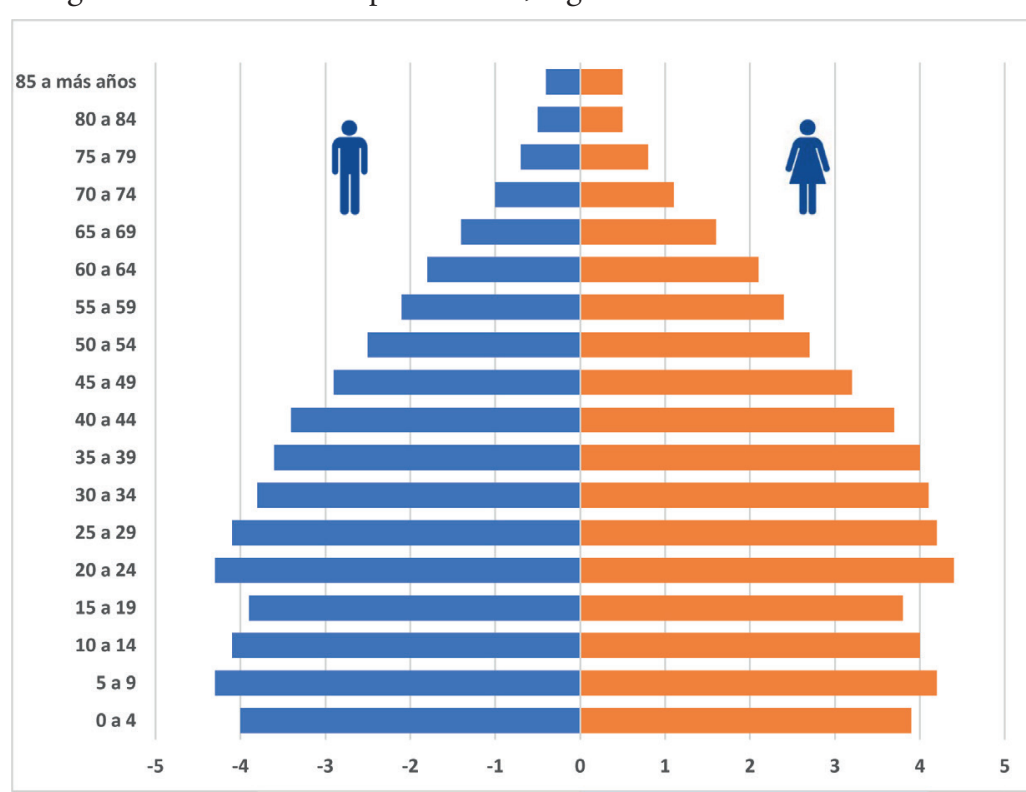

Fuente: INEI - Censos Nacionales 2017; XII Población, VII Vivienda y III de Comunidades Indígenas.

Elaboración propia

Cuadro 3

Región Callao: Distrito donde reside

\begin{tabular}{|c|c|c|}
\hline Distrito & Total & $\%$ \\
\hline Total & 384 & 100.0 \\
\hline Callao & 195 & 50.8 \\
\hline Bellavista & 45 & 11.7 \\
\hline Carmen de la Legua Reynoso & 18 & 4.7 \\
\hline La Perla & 39 & 10.2 \\
\hline La Punta & 4 & 1.0 \\
\hline Mi Perú & 10 & 2.6 \\
\hline Ventanilla & 73 & 19.0 \\
\hline
\end{tabular}

Fuente: Encuesta sobre preferencia turística.

Elaboración propia.

vos, un $18.0 \%$ son cesantes, el $28.4 \%$ son jubilados y un $19.8 \%$ son desocupados (cuadro 6, gráfico 6).

En los resultados en cuanto a si su estado de salud le permite viajar se advierte que el 70.6\% respondió que sí y solo el $29.4 \%$ restante respondió que no (cuadro 7, gráfico 7).

Los datos en cuanto al tipo de turismo que prefiere realizar registran que el $41.7 \%$ prefiere las actividades recreativas, el $36.7 \%$ elige lo cultural, el $8.3 \%$ escoge la salud, el $5.2 \%$ opta por lo vivencial, el $2.3 \%$ selecciona lo gastronómico, por compras o 
Gráfico 3

Región Callao: Distrito donde reside

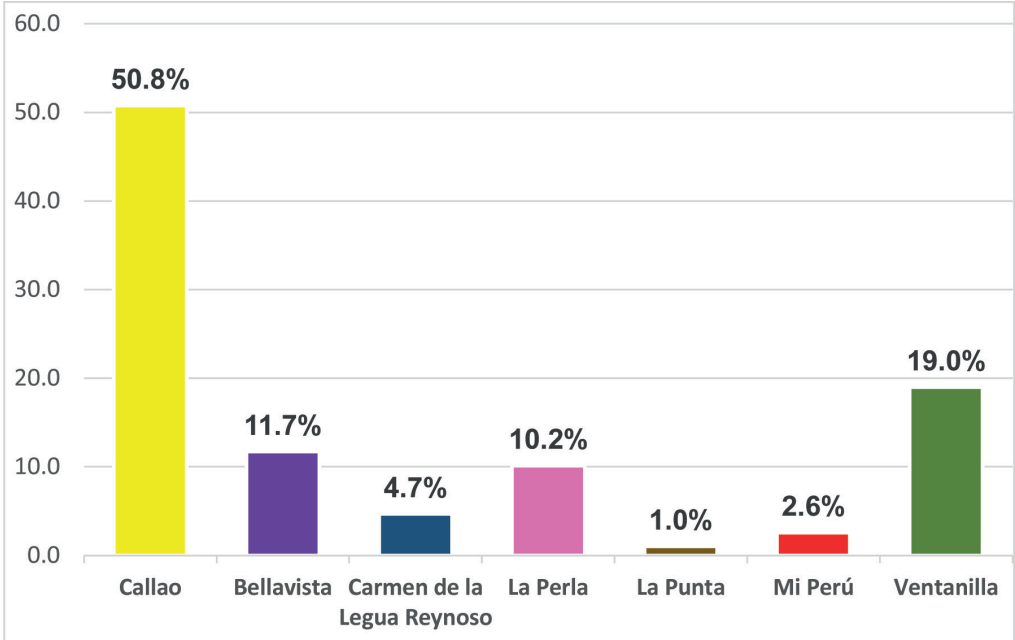

Fuente: Encuesta sobre preferencia turística.

Elaboración propia.

\section{Cuadro 4}

Región Callao: Estado civil de la población adulta mayor

\begin{tabular}{|c|c|c|}
\hline Estado civil & Total & $\%$ \\
\hline Total & 384 & 100.0 \\
\hline Soltero/a & 31 & 8.1 \\
\hline Casado/a & 219 & 57.0 \\
\hline Viudo/a & 82 & 21.4 \\
\hline Divorciado/a & 23 & 6.0 \\
\hline Conviviente & 27 & 7.0 \\
\hline Separado/a & 2 & 0.5 \\
\hline
\end{tabular}

Fuente: Encuesta sobre preferencia turística.

Elaboración propia.

Gráfico 4

Región Callao: Estado civil de la población adulta mayor

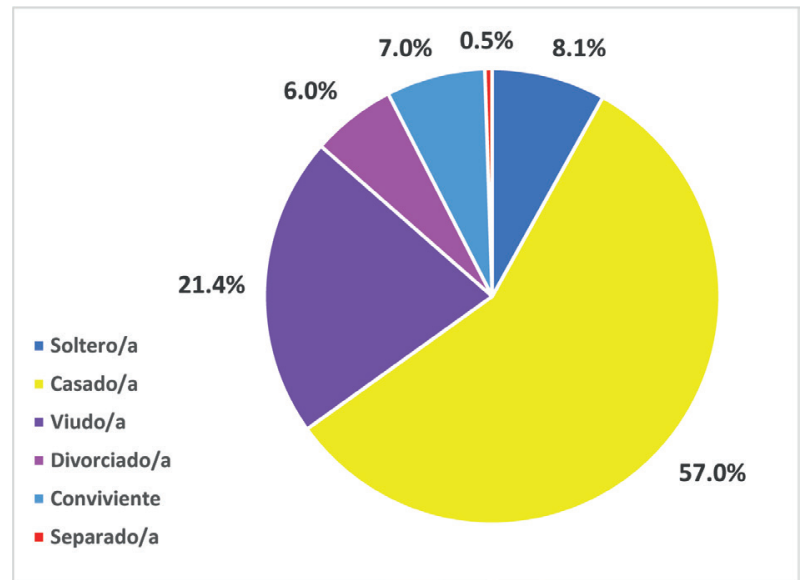

Fuente: Encuesta sobre preferencia turística.

Elaboración propia.
Cuadro 5

Región Callao: Nivel educativo de la población adulta mayor

\begin{tabular}{|c|c|c|}
\hline Nivel educativo & Total & $\%$ \\
\hline Total & 384 & 100.0 \\
\hline Sin nivel & 6 & 1.6 \\
\hline Primaria & 70 & 18.2 \\
\hline Secundaria & 191 & 49.7 \\
\hline $\begin{array}{c}\text { Superior universitaria y no } \\
\text { universitaria }\end{array}$ & 117 & 30.5 \\
\hline
\end{tabular}

Fuente: Encuesta sobre preferencia turística. Elaboración propia.

\section{GRÁFICO 5}

Región Callao: Nivel educativo de la población adulta mayor

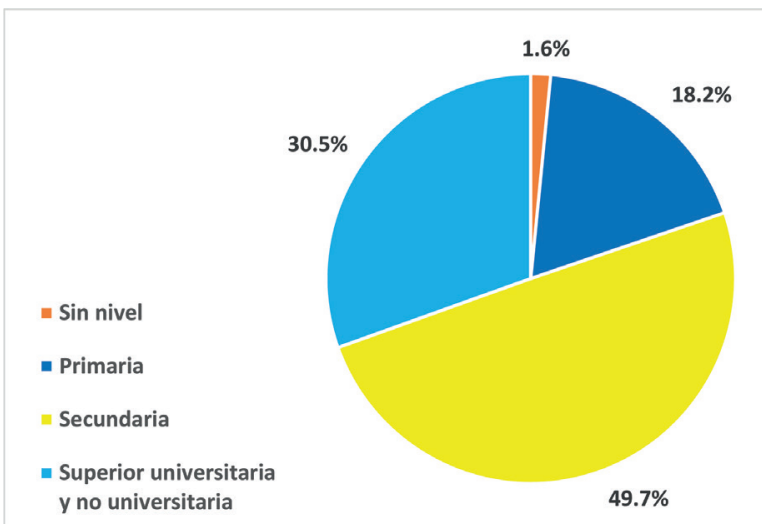

Fuente: Encuesta sobre preferencia turística.

Elaboración propia.

\section{Cuadro 6}

Región Callao: Situación laboral de la población adulta mayor

\begin{tabular}{|c|c|c|}
\hline Situación laboral & Total & $\%$ \\
\hline Total & 384 & 100.0 \\
\hline Trabajador activo & 130 & 33.9 \\
\hline Cesante & 69 & 18.0 \\
\hline Jubilado & 109 & 28.4 \\
\hline Desocupado & 76 & 19.8 \\
\hline
\end{tabular}

Fuente: Encuesta sobre preferencia turística.

Elaboración propia.

simplemente por aventura, y solo el $1.0 \%$ prefiere lo esotérico (cuadro 8, gráfico 8).

Con respecto a la pregunta sobre si usted cree que está en la mejor edad para viajar nos indican que el $71.6 \%$ sí cree que su edad es la más adecuada 


\section{Gráfico 6}

Región Callao: Situación laboral de la población adulta mayor

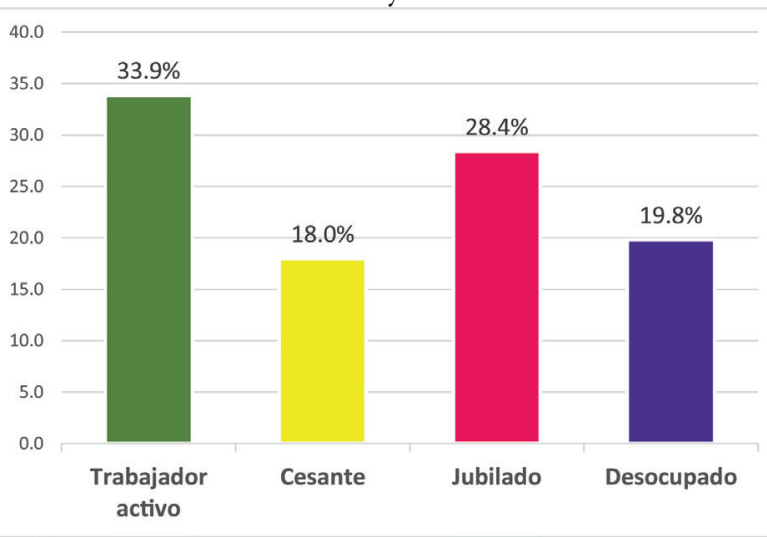

Fuente: Encuesta sobre preferencia turística.

Elaboración propia.

\section{Cuadro 7}

¿Su estado de salud le permite viajar?

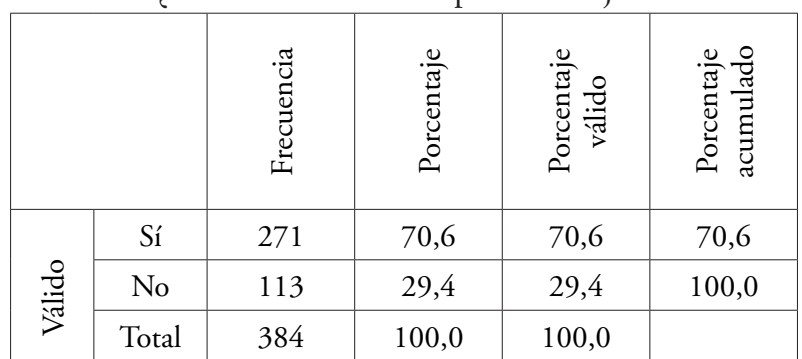

Fuente: Encuesta sobre preferencia turística.

Elaboración propia.

GrÁFICO 7

¿Su estado de salud le permite viajar?

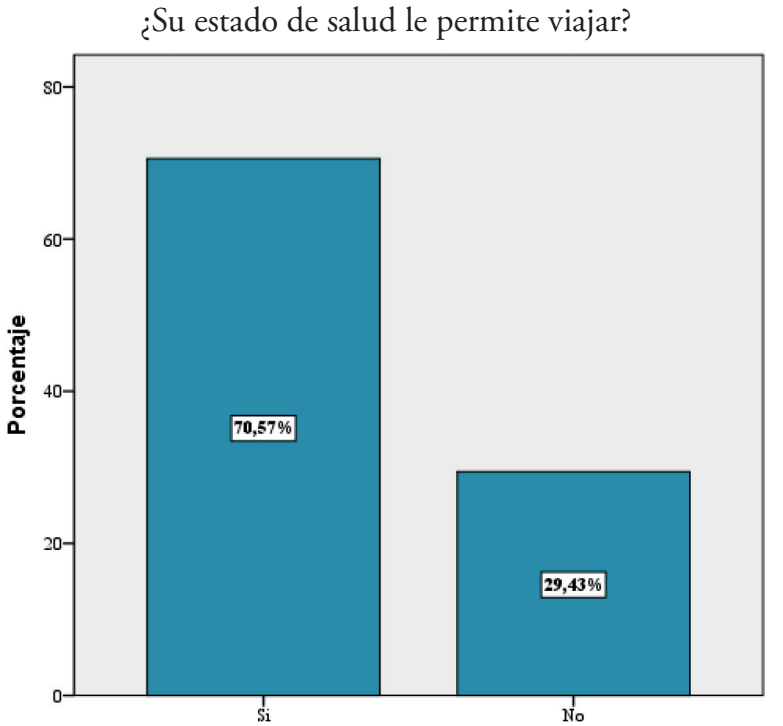

Fuente: Encuesta sobre preferencia turística.

Elaboración propia.
Cuadro 8

El tipo de turismo que usted prefiere realizar es:

\begin{tabular}{|c|c|c|c|c|c|}
\hline & 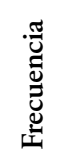 & 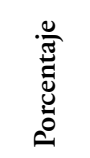 & 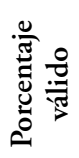 & 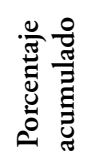 \\
\hline \multirow{9}{*}{$\frac{O}{\stackrel{7}{*}}$} & Cultural & 141 & 36,7 & 36,7 & 36,7 \\
\hline & Recreativo & 160 & 41,7 & 41,7 & 78,4 \\
\hline & Salud & 32 & 8,3 & 8,3 & 86,7 \\
\hline & Compra & 9 & 2,3 & 2,3 & 89,1 \\
\hline & Esotérico & 4 & 1,0 & 1,0 & 90,1 \\
\hline & Vivencial & 20 & 5,2 & 5,2 & 95,3 \\
\hline & Aventura & 9 & 2,3 & 2,3 & 97,7 \\
\hline & Gastronómico & 9 & 2,3 & 2,3 & 100,0 \\
\hline & Total & 384 & 100,0 & 100,0 & \\
\hline
\end{tabular}

Fuente: Encuesta sobre preferencia turística. Elaboración propia.

\section{Gráfico 8}

El tipo de turismo que usted prefiere realizar es:

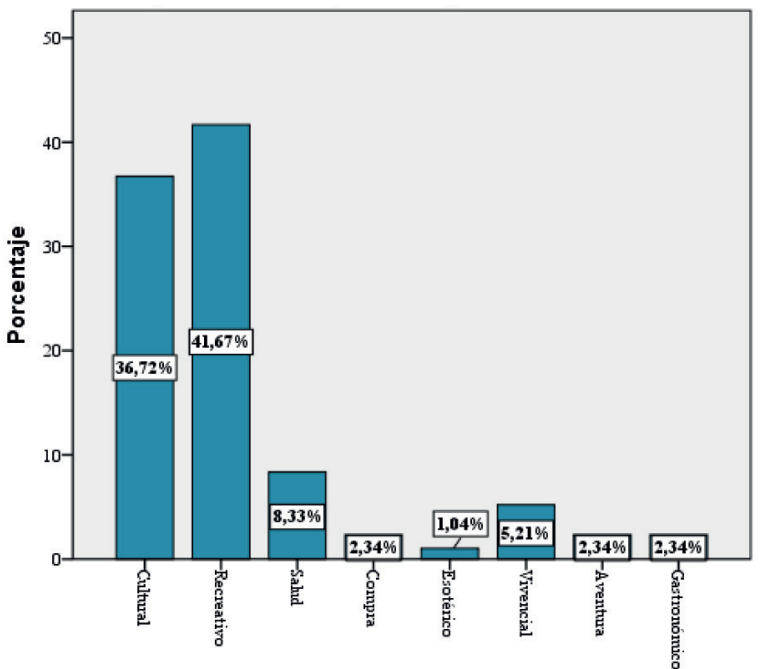

Fuente: Encuesta sobre preferencia turística.

Elaboración propia.

para viajar, en tanto que el $29.4 \%$ considera que su edad no es la adecuada (cuadro 9, gráfico 9).

Los resultados, en cuanto a si considera que hacer un viaje turístico es una excelente forma de emplear su tiempo libre, permiten conocer que el $90.9 \%$ respondió de manera afirmativa, en tanto que el 9.1\% restante respondió en forma negativa (cuadro 10, gráfico 10).

En cuanto a si considera que viajar le produce beneficio a su salud, se aprecia que el $87.2 \%$ sí con- 
Cuadro 9

¿Usted cree que está en la mejor edad para viajar?

\begin{tabular}{|c|c|c|c|c|c|}
\hline & 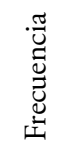 & 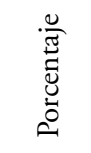 & 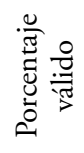 & 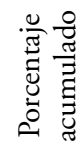 \\
\hline \multirow{3}{*}{$\frac{8}{\frac{7}{3}}$} & Sí & 275 & 71,6 & 71,6 & 71,6 \\
\hline & No & 109 & 28,4 & 28,4 & 100,0 \\
\hline & Total & 384 & 100,0 & 100,0 & \\
\hline
\end{tabular}

Fuente: Encuesta sobre preferencia turística. Elaboración propia.

GRÁFICO 9

¿Usted cree que está en la mejor edad para viajar?

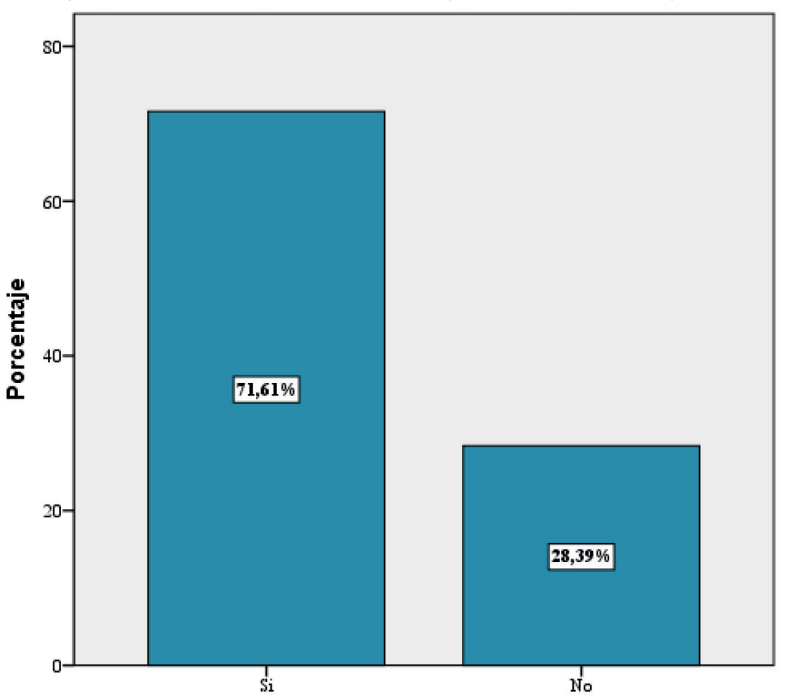

Fuente: Encuesta sobre preferencia turístca. Elaboración propia.

\section{Cuadro 10}

Región Callao: ¿Considera usted que hacer un viaje turístico es una excelente forma de emplear su tiempo libre?

\begin{tabular}{|c|c|c|}
\hline Respuesta & Total & $\%$ \\
\hline Total & 384 & 100.0 \\
\hline Sí & 349 & 90.9 \\
\hline No & 35 & 9.1 \\
\hline
\end{tabular}

Fuente: Encuesta sobre preferencia turística.

Elaboración propia.

sidera que viajar le hace bien a la salud, mientras que el $12.6 \%$ restante considera que los viajes no le hacen bien a la salud (cuadro 11, gráfico 11).

Sobre el ítem si dispone de recursos económicos para viajar, los resultados dan cuenta que el 57\% contestó que sí y el $43 \%$ restante respondió que no
Gráfico 10

Región Callao: ¿Considera usted que hacer un viaje turístico es una excelente forma de emplear su tiempo libre?

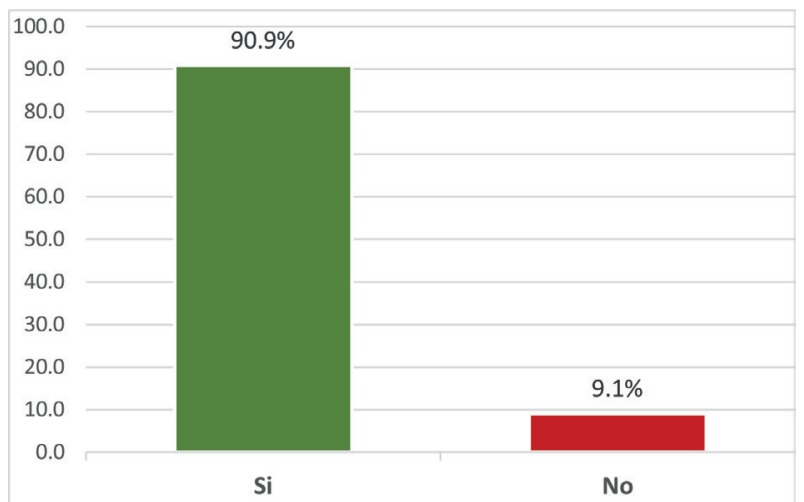

Fuente: Encuesta sobre preferencia turística

Elaboración propia

\section{Cuadro 11}

Región Callao: ¿Considera usted que viajar le produce beneficio a su salud?

\begin{tabular}{|c|c|c|}
\hline Respuesta & Total & $\%$ \\
\hline Total & 384 & 100.0 \\
\hline $\mathrm{Si}$ & 335 & 87.2 \\
\hline $\mathrm{No}$ & 49 & 12.8 \\
\hline
\end{tabular}

Fuente: Encuesta sobre preferencia turística.

Elaboración propia.

\section{GrÁfico 11}

Región Callao: ¿Considera usted que viajar le produce beneficio a su salud?

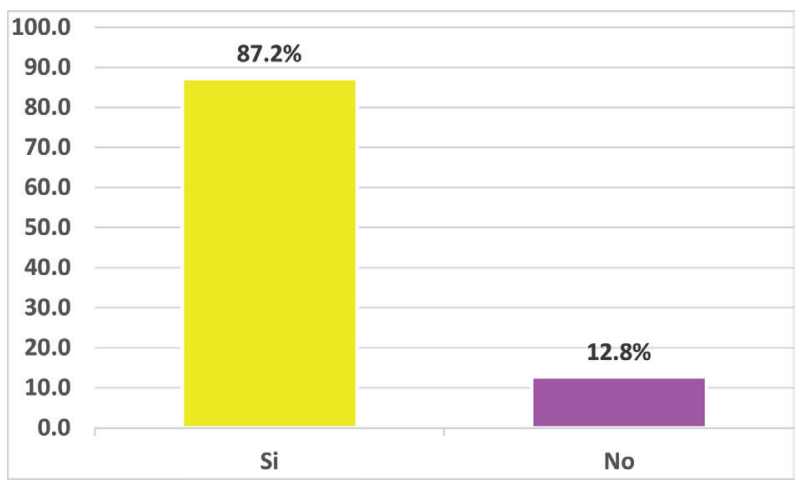

Fuente: Encuesta sobre preferencia turística. Elaboración propia.

(cuadro 12, gráfico 12). Con respecto a la duración de los viajes, las cifras indican que el $30.5 \%$ prefieren los viajes largos y el $69.5 \%$ prefieren los viajes cortos (cuadro 13, gráfico 13). 
Cuadro 12

¿Usted dispone de recursos económicos para viajar?

\begin{tabular}{|c|c|c|c|c|c|}
\hline & 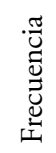 & 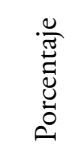 & 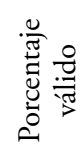 & 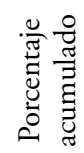 \\
\hline \multirow{3}{*}{ 退 } & Sí & 219 & 57,0 & 57,0 & 57,0 \\
\hline & No & 165 & 43,0 & 43,0 & 100,0 \\
\hline & Total & 384 & 100,0 & 100,0 & \\
\hline
\end{tabular}

Fuente: Encuesta sobre preferencia turística.

Elaboración propia.

\section{Gráfico 12}

¿Usted dispone de recursos económicos para viajar?

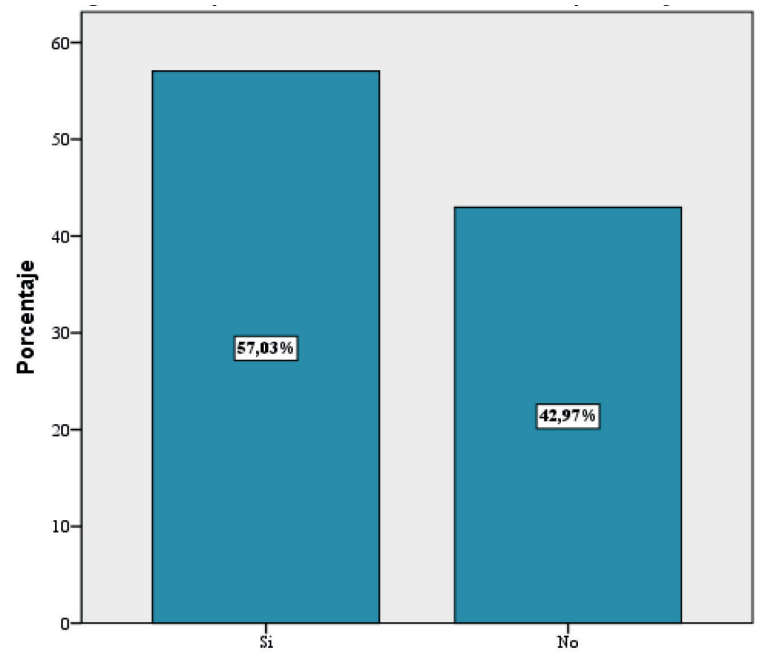

Fuente: Encuesta sobre preferencia turística.

Elaboración propia.

\section{Cuadro 13}

Región Callao: Usted prefiere los viajes largos o cortos

\begin{tabular}{|c|c|c|}
\hline Pregunta & Total & $\%$ \\
\hline Total & 384 & 100.0 \\
\hline Largos & 117 & 30.5 \\
\hline Cortos & 267 & 69.5 \\
\hline
\end{tabular}

Fuente: Encuesta sobre preferencia turística. Elaboración propia.

Los resultados acerca de la cantidad de días que prefiere viajar, señalan que el $56.0 \%$ prefiere 10 días; el 26.0\%, 20 días; el 11.2\%, 30 días y un $6.8 \%$ prefiere una mayor cantidad de días (cuadro 14 , gráfico 14 ).

\section{Gráfico 13}

Región Callao: Usted prefiere los viajes largos o cortos

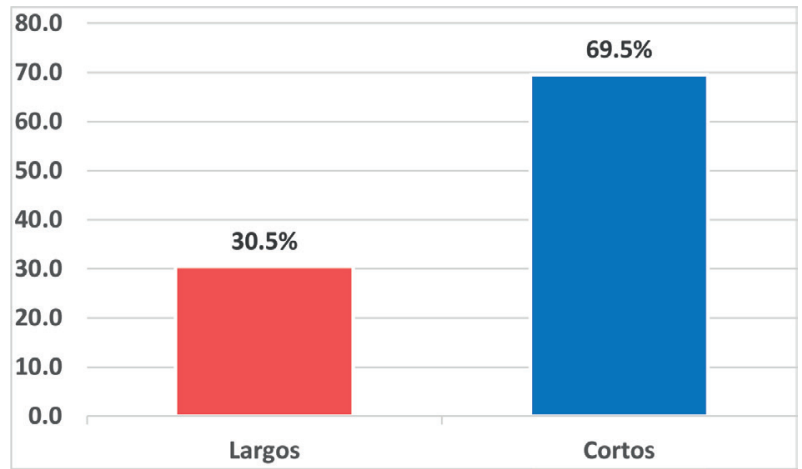

Fuente: Encuesta sobre preferencia turística.

Elaboración propia.

\section{Cuadro 14}

Región Callao: ¿Usted por cuántos días preferiría viajar?

\begin{tabular}{|c|c|c|}
\hline Preguntas & Total & $\%$ \\
\hline Total & 384 & 100.0 \\
\hline 10 días & 215 & 56.0 \\
\hline 20 días & 100 & 26.0 \\
\hline 30 días & 43 & 11.2 \\
\hline Más días & 26 & 6.8 \\
\hline
\end{tabular}

Fuente: Encuesta sobre preferencia turística.

Elaboración propia.

GrÁfico 14

Región Callao: ¿¿Usted por cuántos días preferiría viajar?

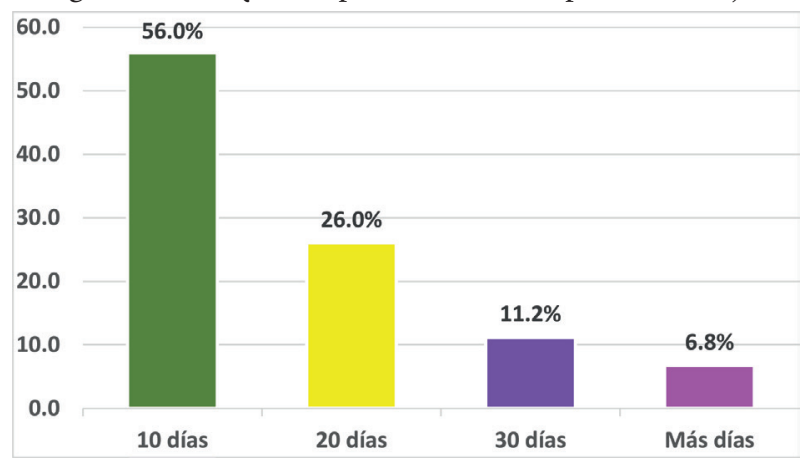

Fuente: Encuesta sobre preferencia turística. Elaboración propia.

\section{Discusión}

Los resultados obtenidos muestran que conforme se incrementa la edad de los adultos mayores, la población disminuye tanto en cifras absolutas como relativas. Esto se observa en el grupo que tiene 85 a más ańos de edad y que está constituido por 8749 perso- 
nas y que representa el $0.9 \%$ de la población total de la región. Esta situación es resultado de la alta tasa de mortalidad, que afecta sobre todo a las personas adultas mayores de sexo masculino. Con relación al sexo, los datos revelan que existen mayor número de personas del sexo femenino (65 647) y menor número de varones $(57510)$, es decir, hay una diferencia entre ambos sexos de 8137 a favor de las mujeres. Este resultado nos indica que las mujeres viven más años que los hombres, es decir, su esperanza de vida es mayor que la de los varones, lo cual se aprecia en el índice de masculinidad, donde se demuestra que hay un exceso de mujeres. El IM de los adultos mayores es de 87.6 hombres por cada 100 mujeres.

En la pirámide poblacional 2017 se observa que en la región Callao se produce el proceso de envejecimiento demográfico, que se hace visible tanto en la base como en la cúspide de la pirámide. La base es estrecha, debido a la disminución de la tasa de natalidad y a un incremento de la población en la parte central de la pirámide, en donde se concentra la población que se encuentra en edad de trabajar; y en la parte superior, a partir del grupo de edad de $60 \mathrm{a}$ más años, se aprecia un incremento significativo de la población adulta mayor, en un $12.4 \%$.

Con relación al estado civil, se aprecia que las personas solteras han alcanzado el mayor porcentaje frente a las personas cuyo estado civil es separado(a). Menos de la cuarta parte de la población lo forman personas viudas y es consecuencia del aumento de la mortalidad y de la disminución de la esperanza de vida que afecta especialmente a los hombres.

Con respecto al nivel educativo alcanzado por los adultos mayores, destacan con mayor porcentaje los niveles primario y superior universitario y no universitario. Esta situación se debe a que en la región existen facilidades para realizar estudios en todos los niveles. Son personas instruidas y por este motivo prefieren realizar turismo cultural.

Los datos de la situación laboral de los adultos mayores muestran que el $33.9 \%$ trabajan y más del $66.0 \%$ son cesantes, jubilados o desocupados. Estos datos nos indica que hay un gran porcentaje de adultos mayores que disponen de tiempo libre y lo podrían utilizar para realizar turismo o actividades recreativas.

Con relación al estado de salud de los adultos mayores, las cifras demuestran que el $70.6 \%$ de las personas están en condiciones de viajar y el $29.4 \%$ manifestaron que su estado de salud no le permite viajar. Según la transición epidemiológica, el patrón de enfermedad y muerte ha cambiado respecto al siglo pasado. La población padece de enfermedades no transmisibles, sufren de hipertensión, diabetes, enfermedades respiratorias, artritis, gastritis, estrés, depresión, entre otras dolencias. Las personas que sufren estas enfermedades crónicas contestaron que su estado de salud sí les permite viajar y además consideran que viajar sí les produce beneficios en su salud y también mejora su calidad de vida. Las personas que contestaron que su salud no les permite viajar fue debido a que padecen enfermedades degenerativas o enfermedades graves como el cáncer en estado avanzado, o personas que tienen impedimentos físicos como caminar, ceguera, entre otras causas.

Los adultos mayores prefieren en primer lugar realizar actividades recreativas (41.7\%) y un $36.7 \%$ prefiere el turismo cultural. Esta situación se debe a que más de la mitad de la población adulta mayor no cuenta con los recursos económicos suficientes para sufragar viajes turísticos, ya que la mayoría de ellos son cesantes o jubilados y sus pensiones son muy bajas. Por este motivo prefieren las acciones recreativas, que se realizan por un tiempo menor de 24 horas y porque no generan mucho gasto. Por otro lado, el $36.7 \%$ de la población prefiere el turismo cultural, que es practicado por personas que han alcanzado el nivel secundario y nivel de educación superior universitario y no universitario. Estas personas eligen visitar museos, monumentos históricos, sitios arqueológicos, etc. Hay que tener en cuenta que ellos sí disponen de los recursos económicos suficientes, que les permiten viajar por varios días. Un $56.0 \%$ prefiere viajar por un lapso de 10 días y otros (6.8\%) optan por viajes largos hasta de 30 días. Es decir que la duración de los viajes está en función de su capacidad económica, de su estado de salud y de su disponibilidad de tiempo libre.

Un gran porcentaje de los adultos mayores (71.6\%) cree que sí están en la mejor edad para viajar, mientras que un $29.4 \%$ considera que no están en la edad adecuada para viajar. Las personas que respondieron que son cesantes y jubiladas, cuyo estado de salud les permite viajar, trasladarse de un lugar a otro o dedicarlo a realizar diferentes actividades recreativas. Por último, el $90.9 \%$ contestó que viajar 
es una excelente forma de emplear su tiempo libre y solo el $9.1 \%$ respondió que no.

\section{Conclusiones}

La región Callao, por su posición geoestratégica en la cuenca del Pacífico Sur, está destinada a convertirse en el centro del comercio internacional y en un centro turístico de gran jerarquía a nivel nacional e internacional.

El turismo es una actividad que produce efectos económicos positivos en los lugares donde se lo promueve. Esta actividad contribuye al desarrollo de la economía en sus diferentes dimensiones. En tal sentido, la región Callao debe seguir fomentando el turismo para favorecer el crecimiento económico y social de la región.

En la región Callao, la población adulta mayor se incrementa constantemente, al igual que la esperanza de vida. Es decir, las personas viven más años. Esta situación se observa en la pirámide poblacional, sobre todo en la parte superior de la pirámide, a partir del grupo de edad de 60 a más años, donde la población crece tanto en cifras absolutas como relativas. Por consiguiente, es necesario incorporar a este segmento de la población a las políticas públicas de turismo y al marketing turístico, favoreciendo económicamente a las empresas turísticas.

Por tal motivo, es importante conocer las preferencias turísticas, los gustos y los deseos de los adultos mayores para elaborar políticas públicas de turismo especializado, orientados para este segmento poblacional, teniendo en cuenta su estado de salud, capacidad económica y el tiempo libre que disponen con la finalidad de sacarlos de su estado de aislamiento y puedan relacionarse con otras personas de su mismo grupo etario, para contrarrestar los efectos nocivos del sedentarismo y procurar aumentar su actividad física.

\section{Referencias bibliográficas}

Acerenza, Miguel (2006). Efectos económicos, socioculturales y ambientales del turismo. México: Editorial Trillas.

Castillo, N. (2018). Experiencias en socialización y gestión turistica en el adul to mayor valente en Lima. Escuela Profesional de Turismo y Hotelería. Lima: Universidad de San Martín de Porres.

Castaño Blanco, José (2005). Psicología social de los viajes $y$ del turismo. Thomson Editores. España.

Cornachione Larrinaga, María (2006). Psicología del desarrollo. Aspectos biológicos, psicológicos y sociales. Editorial Brujas. Argentina.

Cueva, A. (2014). Estudio exploratorio - descriptivo del segmento adulto mayor-su oferta turística y diseño de estrategias de intervención en la ciudad de Quito. Facultad de Ciencias Administrativas. Ecuador.

De la Torre Padilla, Óscar (1980). Turismo, fenómeno social. Fondo de Cultura Económica. México.

Guerrero Gonzalez, Perla; Ramos Mendoza, J. Roberto (2016). Introducción al turismo. México-

Instituto Nacional de Estadística e Informática (2008). Perfil sociodemográfico de la provincia Constitucional del Callao. Lima.

Instituto Nacional de Estadística e Informática (2018). Resultados definitivos de los censos nacionales 2017, XII de Población, VII de Vivienda y III de Comunidades Indigenas. Lima.

López Nórez, María; Llera Pacheco, Francisco; VÁsQuez Ramírez, Patricia (2009). El papel del turismo en la Recreación del Adulto Mayor. México.

Organización de LAS Naciones Unidas (1982). Asamblea mundial sobre envejecimiento. Viena. Austria.

Organización de las Naciones Unidas (2002). Informe de la segunda asamblea sobre envejecimiento. Madrid. España.

Rodríguez Abitia, Sergio (2010). Reflexiones sobre el ocio y el tiempo libre. México: Editorial Trillas.

Ziperovich, Andrés (2006). Turismo y recreación. México: Editorial Trillas. 